\title{
Trayectorias escolares de hijos de familias agrícolas migrantes en Sonora, México
}

\section{School Careers of Children of Migrant Agricultural Families in Sonora, Mexico}

\author{
Teresa de Jesús Rojas Rangel ${ }^{*}$ (D) https://orcid.org/0000-0003-2877-5011
}

\section{Resumen}

El objetivo del artículo es analizar las trayectorias escolares de preescolar, primaria y secundaria de las/los hijos de familias agrícolas migrantes, y cómo los factores de riesgo, relacionados con los contextos socioeconómicos precarios y participación en los patrones de movilidad, influyen en sus recorridos escolares. Para ello se aplicaron cuestionarios e hicieron entrevistas semiestructuradas. Según los resultados, los asentamientos de dichas familias en zonas de atracción contribuyen a una mayor permanencia y continuidad de sus hijos/as en la escuela. En México este estudio es pionero en reconstruir las trayectorias escolares de quienes cursan hasta tercero de secundaria, pues los existentes están centrados en la educación primaria. Se encontró que un porcentaje alto de estudiantes termina la secundaria con trayectorias teóricas ideales y que una determinante fundamental para el logro exitoso de las trayectorias escolares son los arreglos familiares para permanecer en un solo lugar, a fin de que sus hijos/hijas concluyan la educación básica.

Palabras clave: migración rural; jornaleros agrícolas; educación básica; trayectorias escolares; Sonora; México.

Cómo citar: Rojas Rangel, T. J. (2019). Trayectorias escolares de hijos de familias agrícolas migrantes en Sonora, México. región y sociedad, 3 I, el I8I. doi: I0.22 I98/rys20 I 9/3 |/ I I I

* Universidad Pedagógica Nacional. Área (5) Teoría Pedagógica y Formación Docente. Carretera al Ajusco \# 24, colonia Héroes de Padierna, C.P. 14200, alcaldía Tlalpan, Ciudad de México, México. Correo electrónico: tererojas10@yahoo.com

Recibido: 11 de febrero de 2019

Aceptado: 25 de junio de 2019

Liberado: 2 de octubre de 2019

\begin{abstract}
The objective of the article is to analyze the preschool, elementary and junior high school careers of the children of migrant agricultural families, and how risk factors, related to precarious socio-economic contexts and participation in mobility patterns, influence their educational paths. For that purpose, questionnaires were applied and semi-structured interviews were conducted. According to the results, settling of these families in areas of attraction contributes to a greater permanence and continuity of their children in school. In Mexico, this study is a pioneer in reconstructing the school careers of those who attend up to third grade of junior high school, since the existing ones are focused on elementary education. It was found that a high percentage of students complete junior high school with ideal theoretical careers and that an important determinant for the successful achievement of school careers are family arrangements to remain in one place, so that their sons / daughters complete their basic education.

Keywords: rural migration; agricultural day laborers; basic education; school careers; Sonora; México.
\end{abstract}




\section{Introducción}

Sonora es líder en la producción de diversas variedades de uva; en 2017 aportó $84.4 \%$ de las 415885 toneladas generadas en el país. Según estimaciones de la Secretaría de Agricultura, Ganadería, Recursos Hidráulicos, Pesca y Acuacultura (SAGARHPA, 2018), en ese ciclo agrícola se cosecharon 334355 toneladas del fruto, de las que $57.7 \%$ se destinó a la exportación y venta en el mercado estadounidense, lo que representó $\mathbf{4 8 5 . 5}$ millones de dólares en divisas. Dicha producción demanda gran cantidad de fuerza de trabajo, se estima que 100000 jornaleros agrícolas laboran de diciembre a julio (Miranda, 2018), periodo en el que se concentra la cosecha de uva y otros productos agrícolas en la Costa de Hermosillo, Caborca y Guaymas.

La mano de obra requerida se satisface con jornaleros locales, asentados y migrantes - muchos de origen indígena-, que provienen de entidades del centro y sur de México y de municipios de Sonora (Haro, 2007; Lara-Flores y Sánchez-Saldaña, 2015; Minjárez-Sosa, 2014), por lo que algunas regiones del estado se han convertido en polos importantes de atracción de fuerza de trabajo temporal y asentada.

Factores como la pobreza, la falta de trabajo, los salarios bajos y las condiciones precarias de vida, entre otros, asociados con la inseguridad en sus localidades, son las causas principales que obligan a millones de campesinos e indígenas a desplazarse de sus territorios originales a regiones rurales del país con mayor potencial económico, como Sonora; la migración es la única estrategia de sobrevivencia y el medio para garantizar su reproducción biológica y cultural. Tanto en las zonas de origen como en las de atracción, estas familias viven en condiciones precarias, sin acceso a los servicios básicos ni a la protección social, y su vulnerabilidad es alta debido a sus perfiles económicos, sociales y culturales y a la falta de protección gubernamental.

Los trabajadores del campo que arriban a Sonora se desplazan en grupos familiares completos, y sus perfiles sociodemográficos son heterogéneos. De acuerdo con el estudio de Rodríguez-Solera y Rojas-Rangel (2017), realizado con hijos de familias asentadas y migrantes temporales, $41.3 \%$ de los padres reconocen su pertenencia a un grupo étnico, y $28 \%$ es hablante de lengua indígena, en su mayoría de náhuatl. Tienen una escolaridad muy limitada, casi $24 \%$ no sabe leer ni escribir, y 69.6 afirma haber asistido a la escuela y, de dicho porcentaje, 67.5 contaba hasta con cinco años de estudios, 18.9 reportó seis de escolarización acumulada, los que corresponden a la primaria, y 13.5 dijo haber cursado nueve grados, equivalentes a la secundaria.

Las familias agrícolas jornaleras realizan el "trabajo en negro" del proceso productivo, ${ }^{1}$ que no está regulado por las normas laborales ni brinda seguridad social (Labrunée y Gallo, 2005); en esta modalidad, sobre todo los trabajadores

1 Aunque se identifica a una minoría de jefes de familias, principalmente asentadas, que opta por realizar actividades comerciales relacionadas con el jornalerismo temporal, como "raiteros" o la renta de "cuarterías" para el alojamiento de los migrantes temporales, así como otras labores informales (Lara-Flores y Sánchez-Saldaña, 2015). 
temporales están sujetos a flexibilidad laboral alta y a condiciones desfavorables, típicas del trabajo precario: ${ }^{2}$

La mayoría de las tareas se pagan por surco o por planta (mata), mientras que el corte se remunera por caja de uva cortada. De acuerdo a los trabajadores, el corte es la tarea que más les conviene realizar, por los recursos económicos que se pueden obtener, dado los volúmenes de uva que se corta en las tres semanas en las que los empresarios buscan obtener el máximo beneficio con la exportación. En opinión de los cuadrilleros entrevistados, una pareja de trabajadores experimentados puede cortar alrededor de 150 cajas diarias de uva, con un pago de 6 pesos cada una, mientras que un cortador novato puede alcanzar las 40 o 50 cajas diarias (Lara-Flores y Sánchez-Saldaña, 2015, p. 82).

No obstante estas condiciones de trabajo precario, en las últimas décadas en Sonora se observan procesos crecientes de asentamientos residenciales, de quienes en otro momento fueron trabajadores temporales, en las localidades donde se ubican los enclaves agrícolas (Lara-Flores y Sánchez-Saldaña, 2015). Los asentamientos también han dado lugar a relaciones laborales nuevas entre los trabajadores y las empresas agrícolas, en la medida que tienen mayor libertad para elegir la que les conviene y según los salarios que ofrece. Asimismo, algunos de los migrantes asentados han logrado obtener mejores puestos y condiciones laborales, mayor estabilidad económica para los trabajadores y sus familias (Vargas-Evaristo, 2007; Velasco-Ortiz, 2013), y otras formas de organización de los campesinos. Estos procesos de arraigo no solo incluyen los asentamientos residenciales, sino el despliegue de múltiples y complejas estrategias de adaptación e integración social y cultural en las zonas de atracción (Velasco-Ortiz, 2013). También han generado el surgimiento de demandas sociales nuevas, particularmente ante el hacinamiento y la carencia de servicios públicos, tanto en las cuarterías aledañas como en las galeras de las empresas, donde residen muchos de ellos (Haro, 2007; Minjárez-Sosa, 2014). El arraigo en las regiones de recepción posibilita el surgimiento de estrategias nuevas de reproducción familiar. Pero, sobre todo, las dinámicas de asentamiento e integración en las zonas de atracción generan mayores oportunidades educativas para los hijos de los jornaleros asentados.

Al mismo tiempo, en estas regiones de Sonora se siguen observando concentraciones temporales grandes de población flotante, que permanecen durante los ciclos productivos. Son familias campesinas e indígenas provenientes tanto de la entidad como de los estados más pobres del sur del país, que se desplazan de manera pendular o circular hacia Sonora en busca de oportunidades de empleo en la agricultura. Las familias migrantes pendulares, que representan 42.3\% (Rodríguez-Solera y Rojas-Rangel, 2017), migran de acuerdo con el inicio y término de los ciclos de cultivo, y retornan de manera cíclica a sus lugares

2 Para Chong-González, Herrera-Tapia, Chávez-Mejía y Sánchez-Plata (2015), el trabajo precario se caracteriza por: a) la discontinuidad en el tiempo; b) la inexistencia de contratos; c) la falta de respeto al salario mínimo; d) los horarios muy amplios y e) la carencia de seguridad social, entre otros aspectos, que tienen consecuencias negativas para los trabajadores y sus familias. 
de origen por tiempos definidos. Mientras que las familias migrantes circulares o golondrinas, que se estiman en $7.3 \%$, al igual que las pendulares, permanecen en el estado durante el ciclo productivo, para después desplazarse durante temporadas cortas a otras entidades, y postergan hasta por años su retorno a las localidades de origen. Movilidad que incide, de manera negativa y a la vez diferenciada -según sea pendular o circular-, en el acceso, permanencia y continuidad educativa de sus hijos.

\section{Aproximación conceptual}

A partir de la última década han proliferado los estudios acerca del estado que guarda la atención educativa de los hijos de las familias agrícolas migrantes (FAM). Desde distintas aristas de análisis, se da cuenta de los límites de la política educativa para otorgarles una educación de calidad y con equidad, que responda a sus formas de vida, contextos sociales y culturales (Miranda-López, 2015; Miranda-López e Islas-Dossetti, 2015; Rodríguez-Solera, 2007, 2009; Rojas-Rangel, 2011, 2014); de las necesidades y experiencias locales instrumentadas para su atención en varias regiones del país, en contextos agroproductivos diferentes (Leal-Sorcia, 2007; Méndez-Puga, 2012; Méndez-Puga, Castro-Valdomino y Vargas-Garduño, 2018; Ramírez-Iñiguez, 2014; Ramírez-Izúcar y Reyes de la Cruz, 2005; Rodríguez-Solera, 2007; Rojas-Rangel, 2018); de los retos pendientes para brindarles educación pertinente, para que puedan concluir con éxito la educación básica (Albarrán-López y Santos-Bautista, 2008; Lugo-Medina y Medécigo-Shej, 2007; Rojas-Rangel, 2016; Yurén-Camarena, Rojas-Mendoza, de la Cruz-Reyes, Espinosa-Meléndez y Escalante-Ferrer, 2011). Sin embargo, a pesar de esta amplia producción hay pocos trabajos que analizan las trayectorias escolares de los hijos de las FAM (Rodríguez-Solera y Rojas Rangel, 2017; Rojas-Mendoza, 2011; Rojas-Mendoza y Yurén-Camarena, 2011).

Por lo tanto, no se cuenta con información suficiente sobre los procesos de ingreso, egreso, transición y continuidad entre los niveles educativos por los que transitan, y menos aún que explique cómo se entrelazan con los contextos familiares, itinerarios laborales y patrones de movilidad. Esto en la medida en que las trayectorias escolares solo pueden entenderse "[...] en el marco de las complejas interacciones entre condicionantes estructurales y contextuales, las mediaciones institucionales y las estrategias subjetivas puestas en juego por cada individuo" (Toscano, Briscioli y Marrone, 2015).

Las trayectorias escolares, denominadas trayectorias de escolaridad por Rojas-Mendoza y Yurén-Camarena (2011), suponen la idea de movimiento dentro del espacio social de la escolarización. Las autoras hacen referencia al recorrido de las/los alumnos por los grados y niveles escolares de acuerdo con la normatividad establecida. Y, a partir del reconocimiento del papel y la agencia que los alumnos despliegan en los procesos de escolarización, las definen como "[...] la serie de posiciones sucesivas en el sistema escolar que un sujeto recorre a lo largo de su vida. [...] la trayectoria de escolaridad se ma- 
nifiesta como decisiones y comportamiento del sujeto a lo largo de su paso por la escuela y en su transición de un nivel de escolaridad a otro" (2011, pp. 3-4).

Para Terigi, las trayectorias escolares son de dos tipos: a) las teóricas (o ideales), definidas por el sistema educativo que organiza la escolarización por niveles, el currículo por grados y la anualización de los grados de acuerdo con los avances de instrucción: "[...] expresan itinerarios en el sistema que siguen la progresión lineal prevista por este en los tiempos marcados por una periodización estándar" (2007, p. 2) y b) las no encauzadas (o reales), en las que se reconoce la heterogeneidad y contingencias en los modos como los estudiantes realizan sus itinerarios escolares (Terigi, 2007, 2008; Toscano, Briscioli y Marrone, 2015).

En las trayectorias teóricas, el tránsito de los estudiantes por los niveles y grados y los tiempos estipulados para cumplir los procesos de instrucción debe ser lineal, continuo y completo, independientemente de sus diferencias personales, sociales y culturales. Esto significa que cada alumno debe ingresar, permanecer y concluir un grado por año y ciertos grados por nivel, durante la escolarización; normatividad establecida en los modelos educativos uniformantes y homogeneizadores, cuyo criterio básico es tener una edad determinada por grado, con la que el ingreso y egreso está asociada a cada nivel. En México, las/los niños deberían ingresar a preescolar a los tres años cumplidos (al 1 de diciembre del año de inicio del ciclo escolar); a primero de primaria a los seis, y ser menor de 16 para cursar la secundaria en las modalidades regulares (general, técnica y telesecundarias) (SEP, 2016). En esta normatividad no se reconocen las condicionantes familiares ni culturales que propician el ingreso tardío, el abandono temporal o la extraedad. Menos aún que haya decisiones familiares por alargar el ingreso o reingreso de las y los niños a la escuela; razones por las cuales estas trayectorias escolares ideales no se cumplen y se trasforman en reales.

En investigaciones sobre las trayectorias escolares de alumnos que provienen de sectores sociales con mayor desventaja, se han identificado factores que poco favorecen la permanencia y éxito escolar de los estudiantes en la educación básica (Bernardi y Cebolla-Boado, 2014; Blanco-Bosco, Solís-Gutiérrez y Robles-Vásquez, 2014; Casal-Bataller, García-Gracia, Merino-Pareja y Quesada-Felice, 2006; López y Tedesco, 2002; Monarca, Rappoport-Redondo y Fernández-González, 2012). Entre ellos están los relacionados con la demanda educativa (pobreza y la marginación de las familias) y, en general, las condiciones precarias de vida en las que nacen y crecen, y que impiden sus procesos de escolarización (desnutrición, enfermedades diversas, incorporación temprana al trabajo remunerado y doméstico, ambientes de socialización precarios, bajas expectativas y poca participación de los padres de familia en las actividades escolares, la migración y la incorporación al trabajo infantil, entre otros), comparativamente con niñas y niños de otros sectores en mejores posiciones sociales. Por otra parte, la literatura sobre el tema también ha mostrado que existen determinantes asociadas con la oferta educativa que inciden en las trayectorias escolares, entre las más importantes están: a) la poca pertinencia de las políticas y programas educativos: b) la limitada inversión pública en los servicios educativos con los que se les atiende; c) la falta de infraestructura escolar para el trabajo en las aulas; d) la poca formación y bajas expectativas escolares de 
los docentes y, en general, c) la pertinencia en las formas de enseñanza de los profesores. Ambos factores, extraescolares e intraescolares, influyen en la permanencia y continuidad de las y los alumnos durante su tránsito por la educación básica (Monarca, Rappoport-Redondo y Fernández-González, 2012).

Uno de los factores extraescolares, que impactan de manera diferenciada en las trayectorias escolares, son los asentamientos definitivos o temporales y los patrones migratorios (pendular y circular) en los que se inscriben los alumnos. A decir de Velasco-Ortiz, la migración es "[...] un factor de alto costo para la reproducción familiar, en particular para la escolarización de los niños. [...] la condición de ser migrante y vivir en campamentos como indicadores de mayor movilidad geográfica son determinantes para la no escolarización de los niños y niñas [...]" (2013, pp. 212-213).

\section{Notas metodológicas}

La investigación se enfocó en el análisis de las trayectorias escolares, su relación con las formas de asentamiento y los patrones migratorios de las familias de los alumnos inscritos en tercero de secundaria en el Programa de Educación Básica para Niñas y Niños de Familias Jornaleras Agrícolas Migrantes (PRONIM). ${ }^{3}$ Las preguntas que orientaron la investigación fueron: ¿cuáles son las trayectorias escolares de los hijos de las FAM inscritos en tercero de secundaria en el PRONIM en Sonora?; ¿qué características presentan las trayectorias escolares en preescolar, primaria y secundaria de quienes han logrado permanecer en la escuela hasta la secundaria?, y ¿qué diferencias tienen las trayectorias escolares de los alumnos, a partir de los asentamientos y los patrones migratorios de las familias a las que pertenecen?

Por la naturaleza de las preguntas y los objetivos de la investigación, se utilizó una metodología mixta, según el criterio de complementariedad de los métodos cualitativos y cuantitativos (Ortí-Benlloch, 1999; Reese, Kendall y Gallimore, 2013; Sánchez-Gómez, 2015):

La complementariedad hace referencia a las estrategias de integración que incorporan una doble y diferenciada visión de los hechos, la cuantitativa y la cualitativa, donde la una completa la visión de la otra, sin que se produzca solapamiento alguno; representa el grado mínimo de integración de métodos (Sánchez-Gómez, 2015, p. 17).

Para el análisis de corte cuantitativo se optó por una estrategia correlacional o selectiva, mediante la aplicación de la técnica de encuesta en el me-

3 EI PRONIM tuvo cobertura nacional de 2002 a 2013, y a partir de 2014 forma parte del Programa de Inclusión y Equidad Educativa (PIEE). Desde su creación fue diseñado para satisfacer las necesidades educativas de la población infantil jornalera migrante, sobre todo de nivel primaria, mediante la flexibilidad y ajustes del calendario escolar de acuerdo con la movilidad y los periodos de permanencia de los alumnos en las zonas de atracción y de origen, la dosificación de contenidos y el diseño de estrategias didácticas y materiales educativos específicos, así como con la creación de mecanismos menos rígidos de control escolar. 
dio natural que, a decir de Alaminos-Chica y Castejón-Costa “[...] se limita a obtener datos, ordenarlos, clasificarlos y establecer relaciones, no causales, entre variables. Tiene por tanto un carácter predominantemente descriptivo" (2006, p. 24). Para la aproximación cualitativa, se recuperó una perspectiva etnosociológica (Bertaux, 2005), a fin de reconstruir las trayectorias de los actores mediante su propio relato (Bertaux, 2005; Kornblit, 2004). Se utilizó la narrativa porque “[...] ofrece la reconstrucción subjetiva de lo acontecido, esto es, la interpretación de los hechos que jalonan su historia mediante la continua atribución de significados a sus experiencias en la enseñanza, con el establecimiento de relaciones de causalidad entre los hechos [...]" (Bolívar-Botía, Domingo-Segovia y Fernández-Cruz, 2001, p. 44).

Los criterios para la selección de los informantes clave fueron: a) estar inscritos en tercero de secundaria; b) pertenecer a una FAM con antecedentes de desplazamientos; y c) ser integrantes de familias vinculadas con el mercado de trabajo agrícola.

El levantamiento de datos se llevó a cabo en tres centros educativos en los municipios de Hermosillo (campo agrícola El Pañuelo), de Guaymas (campo agrícola Guadalupe) y de Caborca (Viñedos Viva), dispuestos por las autoridades educativas estatales. Dichos centros se ubican dentro de los campamentos agrícolas propiedad de las empresas agroexportadoras - algunos están instalados en vehículos móviles-y, aunque tienen aulas equipadas, no disponen de materiales educativos suficientes, atienden en los turnos matutino y vespertino. Los grupos de secundaria están a cargo de un docente y, en algunos casos, son multigrado. Estas escuelas reciben apoyos alimentarios del Programa de Escuelas de Tiempo Completo de la SEP. Se aplicaron 26 cuestionarios y entrevistas a las/los alumnos durante visitas in situ, en marzo de 2016.

\section{Características de las/los alumnos participantes}

Del total del porcentaje de estudiantes a quienes se les aplicaron los instrumentos para la recopilación de datos, 59 fue de mujeres y 41 de hombres, de 14 a 16 años; 54\% provenía de municipios de Guerrero, 31 había nacido en Sonora, y otro 15 en Veracruz, Michoacán y Sinaloa.

Todos eran hablantes de español (aunque un porcentaje alto afirmó entender alguna lengua indígena a pesar de no hablarla), 7.8\% era bilingüe (náhuatl y español). La mayoría no se autoreconoció como indígena, aunque sabía del origen étnico de sus padres.

Uno de los rasgos más importantes de las/los encuestados es que $92.3 \%$ había cursado el grado más alto de escolarización entre los integrantes de sus familias, indicador que cobra relevancia si se considera la baja escolaridad de sus progenitores, solo $7.7 \%$ pertenecía a un núcleo familiar que había estudiado hasta segundo y tercero de educación media.

En lo que se refiere a la procedencia, $50.4 \%$ de los entrevistados era de familias asentadas, 42.3 pertenecía a las que continuaban inscritas en los desplazamientos pendulares y que, al igual que las asentadas, todas residían en los campos agrícolas, y 7.3 provenía de grupos familiares inscritos en la migración 
circular o golondrina, es decir, que se desplazaban por periodos cortos a otras entidades durante el año, a la vez que regresaban cíclicamente a Sonora durante los ciclos de producción agrícola. En esta trashumancia se identificaron casos de niñas y niños que no había retornado a sus localidades de origen por varios años.

Trayectorias escolares teóricas o ideales y reales no encauzadas

De las/los 26 alumnos participantes en el estudio, 84.6\% tuvo una trayectoria teórica o ideal, equiparable a los itinerarios escolares de cualquiera que cursa la educación básica. Es decir, la mayoría que pertenece a familias agrícolas asentadas y migrantes, que logra inscribirse en tercero de secundaria, ha tenido un recorrido regular en la escuela.

Las y los alumnos con estas trayectorias asistían regularmente a las actividades escolares, y cuando no lo hacían era por periodos breves debido a que estaban enfermos o porque tuvieron que cuidar a algún familiar cercano por la misma causa. Cuando se le preguntó a una de las entrevistadas si faltaba a la escuela, señaló: "Sí, cuando estaba enferma. A veces [...] bueno me enfermé del apéndice. Bueno no me operaron porque pensaron que no tenía eso. Sí, como una semana". Y aunque se identificaron lapsos de dos a seis meses en los que no asistían, debido a la movilidad de las familias migrantes pendulares y circulares, esto no repercutió en sus trayectorias escolares, lo que se explica porque la mayoría (63\%) cursó la primaria y la secundaria (77\%) en Sonora, en el PRONIM. No repitieron curso por falta de documentación o abandono temporal durante los ciclos escolares agrícolas, fueron pocos los casos en los que las/los niños tuvieron que cursar dos veces el mismo grado en preescolar o primaria, entre otras causas debido a que no se les certificó por no tener la edad establecida. Una alumna narró:

Porque salí de cinco años [se refiere a su localidad de origen], iba a cumplir seis cuando iba a entrar a primero de primaria y no me quisieron recibir, y fui de "oyente". Pero sí estudié el primero pero de oyente, porque en la escuela donde estábamos no me querían aceptar los papeles, y ya cuando cumplí los seis años ya me aceptaron. Pero en eso perdí un año, porque yo era la más chiquita (Martha, Escuela Secundaria para Migrantes, Campo Viñedos Viva, Caborca, Sonora, 2016).

Según Terigi $(2007,2011),{ }^{4}$ en lo que respecta a las trayectorias no ideales o “no encauzadas", $15.4 \%$ del total reprobó algún grado de primaria, y 3.5 de secundaria; $80 \%$ de la reprobación se concentró en segundo y tercero de primaria y 20 en segundo de secundaria. Estos porcentajes son altos, en lo que corresponde a la primaria, si se considera que el promedio nacional de reprobación en este nivel para el ciclo 2016-2017 fue de 0.8\% (SEP, 2017, p. 17). Pero en secundaria,

4 Este tipo de trayectoria hace referencia a "[...] las trayectorias escolares reales de los sujetos, podemos reconocer itinerarios frecuentes o más probables, coincidentes con o próximos a las trayectorias teóricas; pero reconocemos también itinerarios que no siguen ese cauce [...] gran parte de los niños y jóvenes transitan su escolarización de modos heterogéneos, variables y contingentes" (Terigi, 2011, p. 3). 
los porcentajes de reprobación están por debajo de la media nacional, estimada en 4.9 para este mismo ciclo (SEP, 2017, p. 19).

Al comparar los porcentajes de reprobación anteriores, hay una diferencia significativa en los resultados, lo que fortalece la hipótesis de que en estos grados de primaria es donde los hijos de las familias migrantes tienen mayores dificultades para transitar por la educación básica. Rojas-Rangel afirma que esta tendencia es nacional:

[...] la matrícula se reduce drásticamente conforme avanzan los grados escolares, sobre todo en la transición del primero al segundo y del segundo al tercer grado. En el tránsito de cuarto a sexto grado se identifica una mayor retención de alumnos, así como menores porcentajes de abandono escolar. La tendencia en la distribución de la matrícula y las trayectorias escolares de los niños, muestra que cuando ellos logran acceder al cuarto grado se presenta un mayor número de probabilidades para permanecer hasta sexto grado (2011, p. 219).

Por otra parte, si se toma como referencia la normatividad de la SEP (2016), que establece los 16 años como la edad máxima para estudiar la secundaria, $11.4 \%$ de estudiantes en situación de extraedad, inscritos en tercero, superan por meses dicha edad, sin llegar a cumplir los 17 años. La mitad de los casos en esta condición provenía de familias migrantes asentadas, no asistieron al preescolar y reportaron ingreso tardío a la primaria y la secundaria, y la otra mitad era de familias migrantes pendulares y menores de edad incorporados al trabajo jornalero, que había enfrentado dificultades para reinscribirse en la escuela en su lugar de origen y reprobado algún grado. Según este testimonio de un informante:

La secundaria la empecé a estudiar aquí, pero no acabé el primer año y fue cuando yo me fui para allá y pues ese año también lo perdí. Allí repetí primero y acá estudié el segundo de secundaria (Franki, Escuela Secundaria para Migrantes, Campo Guadalupe, Guaymas, Sonora, 2016).

Una alumna con extraedad y migrante pendular, que permanecía seis meses en Sonora y regresaba los otros seis a Michoacán, comentó:

Pues allá en Altamirano sí. Cuando estaba en Altamirano sí estudiaba pero donde ya no empecé a estudiar fue en Michoacán porque el papel que nos daban no era válido. Nomás era un papel que nos daban firmado pero no nos lo hacían válido [...]. (Elvira, Escuela Secundaria para Migrantes, Campo Viñedos Viva, Caborca, Sonora, 2016).

La mayoría de los casos en situación de extraedad era de familias migrantes, que habían estado en Sonora desde que tenían 8 o 12 años, y que permanecieron en sus localidades de origen durante sus primeros años de vida, al cuidado de abuelos o parientes cercanos sin tener oportunidad de asistir a preescolar, quizá eso explica la incorporación tardía a los demás niveles de educación básica. 
Como se analizó en otros informes de investigación (Rojas-Rangel, 2011, $2016,2018)$, las causas que pueden explicar las trayectorias escolares no encauzadas de esta población infantil en la primaria son: a) la falta de calidad de los programas educativos, por la carencia de métodos y técnicas didácticas para la enseñanza de la lectoescritura y el español en los primeros grados de primaria; b) las exigencias de adaptación social y cultural que demanda la primaria a niñas y niños con origen étnico y para quienes, en varios casos, este nivel representa su primer acercamiento a la escuela y c) de los 6 a los 12 años es cuando la mayoría reporta sus primeras experiencias laborales (agrícolas y no agrícolas). Al superar estas barreras, una vez inscritos en la secundaria, avanzan de manera regular y continua hasta concluir la educación básica.

\section{El tránsito por los niveles educativos}

En este apartado se presentan, de manera desagregada, las trayectorias por preescolar, primaria y secundaria de los hijos de las FAM, a fin de caracterizar con mayor profundidad las tendencias y reconstruir las experiencias de las y los alumnos durante las etapas de escolarización.

Las trayectorias por preescolar

Un porcentaje de 80.7 asistió a preescolar y 19.3 no tuvo la oportunidad de hacerlo. En el primer caso, hubo gran diversidad en cuanto al número de años cursados; de acuerdo con los datos recopilados, ocho de cada diez niñas y niños que lo hicieron 3.7 cursaron tres años, 2.3 estuvieron dos, y uno asistió solo un año, y de uno de cada diez no se obtuvo información. Otro indicador relevante es que $40 \%$ de ellos nació en Sonora, 80 provenía de FAM asentadas y 20 de familias jornaleras temporales. Aunque cabe señalar que a la mitad de las y los niños de familias asentadas se les llevó a Sonora después de la edad estipulada para cursar preescolar.

Las causas de inasistencia a preescolar son múltiples, una es que, no obstante que en las de destino existen mejores condiciones para asistir a este nivel (Miranda-López e Islas-Dossetti, 2015), hay insuficiencia de servicios educativos en las zonas de origen. Así lo señaló uno de los entrevistados:

No había antes, no había [se refiere a la escuela]. Ya después había no'más primaria, y los maestros, así los pasaban a los de seis años. Los pasaban o ya así a primer grado entraban. El maestro nos apoyaba así porque no había preescolar. O sea los que tenían cinco años iban de oyentes no' más (José, Escuela Secundaria para Migrantes, Campo El Pañuelo, Hermosillo, Sonora, 2016).

Otra de las causas posibles de la inasistencia es que las FAM asentadas y temporales, por su origen étnico, prefieren hacerse cargo de la crianza y cuidado de sus hijos durante los primeros años de vida, o dejarlos con familiares cercanos, como los abuelos (figura parental que aparece con mayor frecuen- 
cia) o con los hermanos mayores, que enviarlos a las instituciones educativas. La investigación de Miranda-López señala que cuando estos menores de edad no asisten a preescolar "[...] el 71.3\% de los niños está con su madre; el 23\% restante pasa el tiempo con distintos miembros de la familia, amigos [...], lo cual significa que las redes sociales y familiares juegan un papel importante [...]" (2015, p. 208). Por lo tanto, las y los niños que no asisten "[...] constituirán una franja de alto riesgo por la debilidad con que se incorporan a la vida familiar y social y, de manera particular, por el déficit de atención educativa que los envuelve" (Miranda-López e Islas-Dossetti, 2015, p. 9).

\section{El trayecto por la primaria}

Para los hijos de las FAM, cursar la primaria presenta el mayor reto y obstáculo por resolver; $69.2 \%$ de todos los/las alumnas tuvieron trayectorias ideales o teóricas mientras que para 30.8 fueron reales o no encauzadas.

Del total de quienes tuvieron trayectorias ideales o teóricas, $75 \%$ cursó preescolar y una cuarta parte no lo hizo, 90 ingresó a la primaria a los seis años y la concluyó a la edad establecida. Los menores nacidos en Sonora representaron $35 \%$ y 45,10 y 5 eran originarios de Guerrero, Veracruz y Sinaloa respectivamente, y 5 de otras entidades federativas.

Las/los estudiantes provenientes de familias asentadas representaron $80 \%$, mientras que 15 eran de las migrantes pendulares, con periodos de asentamiento establecidos, y solo retornaban a sus localidades de origen por un tiempo breve, por lo general en diciembre, para visitar a sus familiares. Por otra parte, $5 \%$ pertenecía a familias migrantes circulares, con desplazamientos a más de un estado o dentro del mismo, pero tenía como centro de arraigo los campamentos en Sonora y la recolecta de uva como fuente de empleo permanente. En cuanto a la incorporación al trabajo, $60 \%$ de los jóvenes reportó haber laborado en la producción agrícola durante los periodos vacacionales, en jornadas matutinas y, en algunos casos, de manera esporádica. Esta es la característica de la mano de obra infantil predominante en Sonora, que favorece la asistencia a la escuela y su continuidad en ella de los hijos de las FAM.

Las trayectorias reales o no encauzadas, de casi 31\% de las/los alumnos, se caracterizaron por ingreso tardío, inasistencias recurrentes, abandono prematuro de las actividades antes de finalizar los ciclos escolares agrícolas, deserción temporal y porcentajes altos de reprobación. En la primaria se identifican más claramente las dificultades que enfrenta la población infantil para permanecer y transitar de manera regular por la escuela. Rojas-Rangel afirma que:

Otro factor importante que dificulta a los niños incorporarse a la escuela es la falta de antecedentes previos a la educación primaria, pues como no tienen experiencia del nivel preescolar presentan resistencias y dificultades para relacionarse e incorporarse a la escuela primaria. Este conflicto llega a constituirse en una causa por la cual muchos niños y niñas se niegan a inscribirse y asistir a la escuela (2011, p. 181). 
En la primaria, las/los niños tienen mayores dificultades para adaptarse a las exigencias y rigidez de las normas escolares, por la edad y etapa de formación. Además, en este nivel comienzan a padecer, de manera más evidente, las carencias y limitaciones del sistema educativo, sobre todo para quienes intentan continuar estudiando en sus localidades de origen durante los periodos de retorno. Al referirse al esfuerzo por asistir a la escuela, un alumno migrante pendular de Guerrero afirmó:

Casi no [iba a la escuela]. Sí iba pero hay veces que allá no iba porque los maestros ni iban a dar clases porque iban de Chilpancingo. Creo sí iban de ahí, y como a veces llovía y así casi no iban en eso (Roberto, Escuela Secundaria para Migrantes, Campo Viñedos Viva, Caborca, Sonora, 2016).

En estas trayectorias fragmentadas, también se puede identificar con claridad la repercusión negativa en el trayecto de la escolarización debido de la movilidad de las familias y la incorporación temprana de los menores de edad al trabajo infantil. Lo que a decir de Velasco-Ortiz serían:

[...] las implicaciones del dominio laboral en la vida cotidiana, particularmente en la fragmentación de la trayectoria escolar de los niños, en gran medida por la movilidad laboral de los padres. [...] cabe reconocer que la inmediatez de la urgencia laboral desplaza de la vida cotidiana otras expectativas o deseos como la escuela de los niños que se ve más como una preocupación externa [...] (2013, p. 203).

Una alumna, con trayectoria escolar discontinua, comentó:

Cuando fuimos la primera vez a la Baja yo tenía diez años. Pues allá, pues nomás trabajaban mi papá y mi mamá y mis dos hermanos y mi hermana Magali. Y pues allá yo no trabajaba porque estaba más chiquita y como no conocíamos porque era la primera vez, pues no salíamos nosotros y como nomás nos quedábamos con mis hermanos los más chiquitos y pues nos la pasábamos allá en el cuarto encerrados [...]. (Aida, Escuela Secundaria para Migrantes, Campo Viñedos Viva, Caborca, Sonora, 2016).

Del total de las/los alumnos con este tipo de trayectoria, $84 \%$ era de familias migrantes pendulares y golondrinas de Guerrero y Michoacán, solo se registró $16 \%$ de las familias asentadas. Por otra parte, la totalidad de niñas y niños con trayectorias no encauzadas tuvo experiencias laborales como jornaleros, algunos desde los nueve años de edad. Según Yurén-Camarena et al. (2011):

[...] quienes provienen de familias jornaleras tienen más dificultades para continuar su trayectoria de escolaridad: para que los jóvenes de una familia jornalera acudan a la escuela secundaria se requiere mantenerse en el mismo lugar después de concluida la zafra y eso significa perder la oportunidad de trabajar en otras cosechas y tener que negociar con el administrador la permanencia en el albergue (p. 9). 
En estos casos se identificaron los porcentajes mayores de reprobación y de ingreso tardío a la primaria y luego a la secundaria, con el efecto consecuente en la extraedad.

\section{El recorrido por la secundaria}

En secundaria, $84.6 \%$ de las/los entrevistados tuvo trayectorias ideales, sin interrupciones ni dificultades para cursar los tres grados; mientras que para $11.5 \%$ de ellos fueron irregulares, ya que había reprobado primero o segundo grado y el abandono temporal fue de $3.9 \% .^{5}$ Quienes reprobaron provenían de familias jornaleras pendulares cuyos hijos enfrentan las consecuencias de la rigidez de los sistemas de control escolar, para continuar sus estudios durante el tiempo que permanecen en sus localidades de origen. Un alumno que vivió esta situación comentó:

Tiempo atrás les habíamos decido [dicho] que les dieran la hoja esa pa' que los inscribieran aquí y no se las querían dar. Y así nos vinimos y no se las dieron, y por eso no nos inscriben a la escuela allá. Porque si nos inscribían ya no nos iban a dar el papel, y a' onde estábamos estudiando y ya no nos inscribieron (José Martín, Escuela Secundaria para Migrantes, Campo Viñedos Viva, Caborca, Sonora, 2016).

Es común que quienes transitan por la educación básica ya no estudian en sus lugares de origen, porque argumentan que "no conocen a nadie", "no tienen amigos" o bien que identifican "su escuela" en las regiones de atracción. Solo hubo dos casos que afirmaron haberse inscrito en la escuela en sus localidades de origen durante septiembre, a principio del ciclo escolar, y que asistieron de forma regular hasta diciembre, mes en el que tienen que emigrar a Sonora.

Las trayectorias de los jóvenes pertenecientes a la migración circular o golondrina son fragmentadas, porque debido a su movilidad permanente no pueden asistir a la escuela de manera regular ni acreditar los ciclos agrícolas escolares, y lo narraron así:

Pues este año no estuve aquí, estaba yo en San Luis. Fue en San Luis, sí porque como de aquí nos fuimos para la Baja y de la Baja nos fuimos para San Luis y de San Luis nos vinimos aquí. En vez de irnos pa' Guerrero nos fuimos pa' la Baja, volvimos a ir apenas. Y ya pues de allá se acabó el trabajo y nos fuimos pa' San Luis. Sí. Y ya de San Luis nos regresamos aquí [...]. Y por eso no fui a estudiar [...]" (José Luis, Escuela Secundaria para Migrantes, campo Viñedos Viva, Caborca, Sonora, 2016).

Los jóvenes de este tipo de familias permanecían en Sonora de diciembre a julio, tres meses en Baja California, de agosto a octubre, de ahí se desplazaban a Sinaloa en noviembre, cuando no retornaban a Guerrero, circuitos que realizaban hasta por tres años continuos. Si bien el porcentaje de familias y, por lo

5 Fue el caso de una menor de edad, hija de una familia asentada, que inició su vida en pareja y quedó embarazada, pero después se reincorporó a las actividades escolares. 
tanto, de alumnas/os identificados en movilidad permanente es mínimo, en comparación con la proporción de las familias jornaleras asentadas y pendulares, la problemática educativa que muestran los hijos de las familias migrantes circulares o golondrinas es la más compleja y difícil de resolver debido, entre otras causas, al poco tiempo que permanecen en las zonas de atracción y en las localidades de origen. En este sentido, la inestabilidad geográfica y residencial es un factor determinante que define sus trayectorias escolares. Según Velasco-Ortiz:

[...] la escolarización es un proceso accidentado, marcado por incorporaciones tardías, suspensiones, rezagos y deserciones, en una dinámica acorde con los avatares de su movilidad geográfica y del empleo también inestable. La marca de la reproducción social es la inestabilidad, por lo que lograr cierta estabilidad y certidumbre absorbe la energía vital de los miembros de la familia, y en esa medida parece que los pequeños logros escolares, como concluir un ciclo o mantenerse en la escuela aun con muy bajos rendimientos, se inscriben en proyectos de vida familiar donde la estabilidad residencial parece un logro importante en un horizonte de muy corto plazo (2013, p. 213).

A pesar de las consecuencias que ocasiona la movilidad de las familias en quienes cursan la secundaria, esto se compensa con el hecho de que $80.7 \%$ del total de las/los alumnos había cursado los tres grados, en los campos agrícolas donde fueron encuestados. La mayoría afirmó que concluyó la secundaria y, en general, toda la educación básica en el PRONIM, impartido por la SEP, cuya flexibilidad permite empatar los calendarios escolares con los ciclos productivos agrícolas en cada entidad federativa (Pacheco-Ladrón de Guevara, Cayeros-López y Madero-Pacheco, 2016). Al analizar la importancia del capital escolar en las trayectorias educativas de jóvenes jornaleros migrantes, Rojas-Mendoza y Yurén-Camarena señalan que un factor que incide en las trayectorias escolares no encauzadas es que estudiaron en escuelas con un programa diferente al PRONIM, que "[...] no están favoreciendo la multiculturalidad y, lejos de ello, constituyen espacios donde se sigue discriminando la lengua y la cultura indígena" (2011, p. 8).

Al referirse a los contextos educativos de las/los niños de las FAM que han logrado cursar la educación básica, Rodríguez-Solera señala que:

[...] los alumnos contaron con buenas condiciones de disponibilidad [hay escuelas en todos los campos] accesibilidad [los migrantes pueden asistir y permanecer en ellas] y adaptabilidad, en tanto han sido flexibles para atender las condiciones particulares de los migrantes, lo cual permitió que niñas y niños asistieran a la escuela desde el nivel preescolar y tuvieran una trayectoria escolar estable durante la primaria y la secundaria (2017, p. 7).

Si bien en esta investigación se coincide con Velasco-Ortiz cuando afirma que "[...] la condición del niño migrante, es decir, la de haber nacido en el estado o fuera de él, es un factor de gran peso en las posibilidades de no asistir a la escuela, posiblemente debido a la inestabilidad residencial asociada a la mi- 
gración" (2013, p. 198), se pudo constatar que un factor positivo para que los jóvenes logren trayectorias escolares regulares son los arreglos familiares de migrantes pendulares y circulares, para que puedan permanecer en los albergues en Sonora durante los ciclos escolares agrícolas, no obstante que los padres o hermanos mayores se desplacen a otras entidades.

Las familias pendulares tienen periodos de asentamiento temporales por lapsos establecidos y solo retornan a sus localidades de origen por un tiempo breve, para visitar a sus familiares, por lo general en diciembre, y en muchos casos sin sus hijos. Por otra parte, el centro de arraigo de las familias migrantes circulares o golondrinas con mayores desplazamientos de un estado a otro, o dentro del mismo, son los campamentos agrícolas en Sonora y la recolecta de uva es su fuente de empleo permanente; y, si bien continúan con su trashumancia, muchas hacen esfuerzos por regresar cada año a Sonora a fin de que sus hijos continúen sus estudios. Esta es una de las causas que explican los asentamientos definitivos de las familias, y se ha convertido en uno de los determinantes extraescolares principales, que favorece el tránsito exitoso de las y los niños por la educación básica.

\section{Reflexiones finales}

A pesar de la precariedad de los contextos económicos y sociales y las condiciones adversas en las que los hijos de las FAM cursan la educación básica, los hallazgos de esta investigación, realizada en tres centros educativos del PRONIM en Sonora, muestran avances importantes en los resultados escolares de quienes provienen de estas familias. Y permiten afirmar que en Sonora las dinámicas de asentamiento definitivo y temporal, así como los arreglos de las familias pendulares y circulares o golondrinos, para disminuir los desplazamientos de sus hijos, están produciendo un efecto significativo en la escolaridad de ellos. En coincidencia con lo afirmado por Lara-Flores y Sánchez-Saldaña, y “[...] a diferencia de cuando se inició la migración de jornaleros en esta y otras regiones del noroeste -momento en el que prevalecían las familias pobres, con niños, todos trabajando en los surcos, la mayoría analfabeta que no hablaba español-, en este caso una buena parte de esos jóvenes tienen al menos estudios de primaria y algunos de secundaria" (2015, pp. 88-89).

Estas aseveraciones se fundamentan en los hallazgos siguientes: a) en los/ las alumnas que logran cursar hasta tercero de secundaria existe un alto porcentaje con trayectorias teóricas ideales, lo que contrasta notoriamente con las fragmentadas, discontinuas e incompletas de la mayoría de niñas/os jornaleros migrantes; b) el obstáculo principal es el trayecto por la primaria, y que una vez que las/los hijos de las FAM logran inscribirse en secundaria, transitan por ella con altas posibilidades de concluirla y c) la existencia de arreglos familiares de migrantes pendulares y golondrinos para permanecer el tiempo suficiente 0 retornar a un lugar, incluso al mismo albergue agrícola, para que sus hijos concluyan la educación básica, aunque las familias se desplacen temporalmente a trabajar a otras entidades. 
Aunque en este estudio los resultados favorables, que muestran las trayectorias escolares de hijos de las FAM, no se pueden explicar solo por los determinantes asociados con los asentamientos y las dinámicas de movilidad, es un factor que influye en buena medida en que sus trayectorias escolares no sean interrumpidas, y que al transitar por la educación básica solo enfrenten las dificultades de cualquier otro/a niña de origen rural. De igual manera, en los logros en las trayectorias escolares se debe reconocer que la mayoría de los/ las alumnas de familias asentadas, migrantes pendulares y golondrinas cursaron preescolar, primaria y secundaria en los servicios educativos del PRONIM en el mismo campamento donde fueron entrevistados, sin que se registre asistencia alta en las escuelas de sus comunidades de origen. A dichos estudiantes se les ha denominado "los hijos del PRONIM", en términos de la escolaridad básica. Lo que se considera determinante, y que contribuyó para que concluyeran la educación básica, a pesar de las dificultades de sus trayectorias escolares, las que al fin de cuentas son reales de niñas y niños destinados al fracaso escolar.

Además, el efecto positivo se observa en las trayectorias escolares de los menores de edad y también en términos intergeneracionales, ya que la escolaridad de las/los alumnos que han concluido la educación básica es un avance importante en la escolaridad de los padres de familia. Un fenómeno que, a decir de Rojas-Mendoza y Yurén-Camarena (2011), representa un empoderamiento de las nuevas generaciones de los hijos de las FAM y un progreso en la construcción de su ciudadanía.

Los hallazgos de esta investigación son significativos, debido a que no existen estudios en el país cuyo objeto de análisis específico sea: a) la reconstrucción de las trayectorias escolares de los hijos de las familias jornaleras migrantes que logran permanecer en la educación básica hasta tercero de secundaria, quienes en el contexto del jornalerismo serían las/los alumnos exitosos en términos de la continuidad educativa y la coincidencia entre el grado que cursan y la edad; b) el análisis de los resultados de las trayectorias escolares de los jóvenes de las FAM durante la secundaria, este estudio ofrece un aporte pionero, ya que la mayoría de investigaciones sobre la escolarización de los hijos de las FAM se han centrado en la primaria y más recientemente en la educación preescolar y c) quizá la aportación más importante, es el segundo estudio, después del de Velasco-Ortiz (2013), que explora los procesos educativos de niñas y niños en el marco de los asentamientos crecientes de las familias jornaleras agrícolas en las regiones del noroeste, como es el caso de Sonora.

\section{Referencias}

Albarrán-López, B., y Santos-Bautista, H. (2008). Los niños migrantes y el derecho a la educación. En D. Cienfuegos, J. Morales y H. Santos (coords.), La migración en perspectiva: fronteras, educación y derecho (pp. 21-50). Chilpancingo: Gobierno del Estado de Guerrero/Secretaría de Asuntos Indígenas/ El Colegio de Guerrero/Universidad Pedagógica Nacional (UPN). 
Alaminos-Chica, A., y Castejón-Costa, J. L. (2006). Elaboración, análisis e interpretación de encuestas, cuestionarios y escalas de opinión. Alcoy: Instituto de Ciencias de la Educación-Vicerectorado de Calidad y Armonización Europea-Universidad de Alicante.

Bernardi, F., y Cebolla-Boado, H. (2014). Clase social de origen y rendimiento escolar como predictores de las trayectorias educativas. RIES. Revista Española de Investigaciones Sociológicas, 146, 3-22. Recuperado de http://ih-vm-cisreis.c.mad.interhost.com/REIS/PDF/REIS_146_011397045219900.pdf

Bertaux, D. (2005). Relatos de vida. Perspectiva etnosociológica. Barcelona: Ediciones Bellaterra, S. A.

Blanco-Bosco, E., Solís-Gutiérrez, P., y Robles-Vásquez, H. (2014). Caminos desiguales. Trayectorias educativas y laborales de los jóvenes en la Ciudad de México. México: El Colegio de México, Instituto Nacional para la Evaluación de la Educación.

Bolívar-Botía, A., Domingo-Segovia, J., y Fernández-Cruz, M. (2001). La investigación biográfico-narrativo en educación. Enfoque y metodología. Madrid: La Muralla.

Casal-Bataller, J., García-Gracia, M., Merino-Pareja, R., y Quesada-Felice, M. (2006). Itinerarios y trayectorias. Una perspectiva de la transición de la escuela al trabajo. Paradigmas laborales a debate. Trayectorias, VIII(22), 9-20. Recuperado de http: / /www.redalyc.org/articulo.oa?id=60715249003

Chong-González, E. G., Herrera-Tapia, F., Chávez-Mejía, C., y Sánchez-Plata, F. (2015). Mercado de trabajo rural y precarización: nuevas condiciones socioeconómicas en el sur del Estado de México. región y sociedad, 27(63), 155-149. doi: 10.22198/rys.2015.63.a240

Haro Encinas, J. A. (2007). Globalización y salud de los trabajadores: jornaleros agrícolas y producción de la uva en Pesqueira, Sonora. región y sociedad, 19(40), 73-105. doi: 10.22198/rys.2007.40.a539

Kornblit, A. L. (2004). Historia y relatos de vida: una herramienta clave en metodologías cualitativas. En A. Kornblit (coord.), Metodologías cualitativas en ciencias sociales. Modelos y procedimientos de análisis (pp. 15-33). Buenos Aires: Editorial Biblos.

Lara-Flores, S. M., y Sánchez-Saldaña, K. (2015). En búsqueda del control: enganche e industria de la migración en una zona productora de uva de mesa en México. En A. Riella y P. Mascheroni (comps.), Asalariados rurales en América Latina (pp. 73-94). Buenos Aires: Universidad de la República/Consejo Latinoamericano de Ciencias Sociales. Recuperado de http://biblioteca.clacso. edu.ar/clacso/gt/20151229035324/AsalariadosRuralesEnAmericaLatina.pdf

Leal-Sorcia, O. (2007). Familia y escuela en comunidades de jornaleros migrantes. Revista Intercontinental de Psicología y Educación, 9(1), 49-66. Recuperado de http: / /www.redalyc.org/pdf/802/80290103.pdf

Lebrunée, M. E., y Gallo, M. E. (2005). Informalidad, precariedad y trabajo en negro: distinción conceptual y aproximación empírica. Realidad Económica, 210, 60-76. Recuperado de http://nulan.mdp.edu.ar/397/1/labrunee-gallo-2005.pdf 
López, N., y Tedesco, J. C. (2002). Las condiciones de educabilidad de los niños y adolescentes en América Latina. Buenos Aires: IIPE. Recuperado de https: / / www.buenosaires.iiep.unesco.org/sites/default/files/educabilidad.pdf

Lugo-Medina, M. I., y Medécigo-Shej, G. A. (2007). Aspectos jurídicos de la educación a niños trabajadores migrantes. Memoria del Congreso Nacional de Investigación Educativa. Mérida: Consejo Mexicano de Investigación Educativa/ Universidad Autónoma de Yucatán. Recuperado de http://www.comie.org. mx/congreso/memoriaelectronica/v09/ponencias/at12/PRE1178258573.pdf

Méndez-Puga, A. M. (2012). Jornaleros agrícolas en Michoacán: un estudio de las necesidades educativas de la población. Reseñas de investigación. Distrito Federal: Consejo Nacional de Ciencia y Tecnología (CONACYT)/Secretaría de Educación Pública.

Méndez-Puga, A. M., Castro-Valdomino, I. L., y Vargas-Garduño, M. L. (2018). La vida de familias jornaleras agrícolas migrantes en campos y escuelas de Michoacán. Desde la mirada infantil. En C. Rodríguez y T. Rojas (coords.), Migración interna, infancia y derecho a la educación. Aproximaciones interdisciplinarias, actores y propuestas de políticas públicas (pp. 181-202). Ciudad de México: Universidad Iberoamericana.

Minjárez-Sosa, L. B. (2014). Exclusión en salud reproductiva; una realidad emergente en contextos rurales de migración indígena. Caso: jornaleras agrícolas en la Estación Pesqueira, Sonora. En J. Enríquez, M. Guillén, B. Valenzuela y M. Jaime (coords.), Sociedad, cultura y educación en Sonora. Problemas, vulnerabilidad y cambio social (pp. 67-94). Hermosillo: Universidad de Sonora. Recuperado de https://sociologia.unison.mx/wp-content/uploads/2019/02/ desierto.pdf

Miranda, M. (2018). Llegan a Sonora 100 mil jornaleros eventuales cada año. Uniradio Noticias. Recuperado de https://www.uniradionoticias.com/noticias/sonora/538374//legan-a-sonora-100-mil-jornaleros-eventuales-cada-ano.html

Miranda-López, F. (2015). Preescolares migrantes. Diagnóstico y propuestas de política pública. Ciudad de México: Editorial Praxis.

Miranda-López, F., e Islas-Dossetti, J. M. (2015). Migración infantil y políticas públicas. El caso de la situación de las niñas y niños en edad preescolar, hijos de jornaleros agrícolas migrantes. XIII Congreso Nacional de Investigación Educativa. México: Consejo Mexicano de Investigación Educativa. Recuperado de http: / / www.comie.org.mx/congreso/memoriaelectronica/v13/doc/1166. pdf

Monarca, H., Rappoport-Redondo, S., y Fernández-González, A. (2012). Factores condicionantes de las trayectorias escolares en la transición entre enseñanza primaria y secundaria. REOP. Revista Española de Orientación Pedagógica, 23(3), 49-62. Recuperado de http://www2. uned.es/reop/pdfs/2012/23-3\%20 -\%20Monarca.pdf

Ortí-Benlloch, A. (1999). La confrontación de modelos y niveles epistemológicos en la genésis e historia de la investigación social. En M. Delgado y J. Gutiérrez (coords.), Métodos y técnicas cualitativas de investigación en ciencias sociales (pp. 87-99). Madrid: Editorial Síntesis/Psicología. 
Pacheco-Ladrón de Guevara, L. C., Cayeros-López, L. I., y Madera-Pacheco, J. A. (2016). Interculturalidad y derecho a la educación de la niñez indígena jornalera migrante. Revista LiminaR. Estudios Sociales y Humanísticos, XI(1), 92105. Recuperado de http://www.scielo.org.mx/pdf/liminar/v14n1/v14n1a7. pdf

Ramírez-Iñiguez, A. (2014). La educación escolar como factor de inclusión social desde un planteamiento sistémico. El caso de la niñez jornalera migrante en los valles de Culiacán, Sinaloa (México) (tesis doctoral). Barcelona: Universidad de Barcelona.

Ramírez-lzúcar, C., y Reyes de la Cruz, V. (2005). La niñez jornalera: educación y trabajo. Oaxaca: CONACYT /Universidad Autónoma "Benito Juárez" de Oaxaca.

Reese, J. L., Kendall, K., y Gallimore, R. G. (2003). Cuantitativos y cualitativos, no cuantitativos vs. cuantitativos. En R. Mejía y S. Sandoval (coords.), Tras las vetas de la investigación cualitativa. Perspectivas y acercamientos desde la práctica. México: Instituto Tecnológico y de Estudios Superiores de Occidente.

Rodríguez-Solera. C. (2007). La educación de menores jornaleros migrantes en el estado de Hidalgo. Reseñas de investigación en educación básica. México: SEP.

Rodríguez-Solera, C. (2009). La educación a niñas y niños jornaleros migrantes en México. Revista EN. Escuela Normal “Miguel F. Martínez" Centenaria y Benemérita. Año 2. Monterrey. Recuperado de http:/ / prug.espacios.net.mx/ wcc-enmfm/sitio/archivos/3_w_RevistaEN_2y3.pdf

Rodríguez-Solera, C. (2017). Características del contexto socioeconómico y educativo de niñas y niños de familias de jornaleros migrantes que han logrado cursar la educación básica en Sinaloa. XIV Congreso Nacional de Investigación Educativa, San Luis Potosí, México. Recuperado de http://www.comie.org. $\mathrm{mx} /$ congreso/memoriaelectronica/v14/doc/0880.pdf

Rodríguez-Solera, C., y Rojas-Rangel, T. (2017). El éxito escolar en condiciones adversas. Relatos de vida y trayectorias escolares de hijos de jornaleros agrícolas migrantes. Manuscrito inédito. Universidad Iberoamericana, Ciudad de México.

Rojas-Rangel, T. (2011). Inequidades. La educación primaria de niños y niñas jornaleros agrícolas migrantes. México: UPN.

Rojas-Rangel, T. (2014). Exclusión y política social para la atención de los jornaleros agrícolas migrantes. México: UPN.

Rojas-Rangel, T. (2016). Logros y retos pendientes para la educación básica de las y los niños agrícolas migrantes: recuento de más de una década de esfuerzos del PRONIM. Revista Universitaria Educa, 5(20). Recuperado de http:// educa.upnvirtual.edu.mx/index.php/memoria/36-revista-20

Rojas-Rangel, T. (2018). Las oportunidades educativas de los niños jornaleros migrantes en Hidalgo. México: UPN.

Rojas-Mendoza, A. (2011). Trayectorias de escolaridad de los jóvenes jornaleros migrantes. El caso de estudiantes egresados del PRONIM en el estado de Morelos (tesis de maestría). Cuernavaca: Universidad Autónoma del Estado de Morelos. 
Rojas-Mendoza, A. , y Yurén-Camarena, T. (2011). Trayectorias de escolaridad de los jóvenes jornaleros migrantes. El caso de estudiantes egresados de escuelas del PRONIM en el estado de Morelos. XI Congreso Nacional de Investigación Educativa. México. Recuperado de http://www.comie.org.mx/congreso/memoriaelectronica/v11/docs/area_12/2412.pdf

Sánchez-Gómez, M. C. (2015). La dicotomía cuantitativo-cualitativo: posibilidades de integración y diseños mixtos. Campo Abierto. Revista de Educación. 11-30. Recuperado de https://mascvuex.unex.es/revistas/index.php/campoabierto/article/view/1679

Secretaría de Agricultura, Ganadería, Recursos Hidráulicos, Pesca y Acuacultura (SAGARHPA). (2018). ¿Sabías que? Sonora es líder en la producción nacional de uva. México. Recuperado de http://oiapes.sagarhpa.sonora.gob.mx/notas/econo/uva.pdf

Secretaría de Desarrollo Social (SEDESOL). (2010). Encuesta Nacional de Jornaleros Agrícolas, 2009. México: SEDESOL.

Secretaría de Educación Pública (SEP). (2016). Preescolar. Primaria. Secundaria. Ciudad de México. Recuperado de https://www2.sepdf.gob.mx/que_hacemos/secundaria.jsp

Secretaría de Educación Pública (SEP). (2017). Principales cifras del Sistema Educativo Nacional 2016-2017. Ciudad de México: Dirección General de Planeación Programación y Evaluación Educativa. Recuperado de https://www. planeacion.sep.gob.mx/Doc/estadistica_e_indicadores/principales_cifras/ principales_cifras_2016_2017_bolsillo.pdf

Terigi, F. Z. (2007). Los desafíos que plantean las trayectorias escolares. III Foro Latinoamericano de Educación. Jóvenes y docentes. La escuela secundaria en el mundo de hoy. Buenos Aires: Fundación Santillana. Recuperado de http:// www.ieo.edu.ar/promedu/trayescolar/desafios.pdf

Terigi, F. Z. (2008). Los cambios en el formato de la escuela secundaria argentina: por qué son necesarios, por qué son tan difíciles. Revista Propuesta Educativa 29, 17(29), 63-71. Recuperado de http:/ / ciequilmes.com/wp-content/ uploads/2013/03/TERIGI-LOS-Cambios-en-el-formato-de-la-escuela-sec..pdf

Terigi, F. Z. (2011). En la perspectiva de las trayectorias escolares. Lecturas posibles sobre el capítulo 3. En Atlas de las Desigualdades Educativas en América Latina. Buenos Aires: Organización de las Naciones Unidas para la Educación, la Ciencia y la Cultura, sede regional Buenos Aires-Organización de Estados Iberoamericanos.

Toscano, G., Briscioli, B. y Marrone, A. (2015). Trayectorias escolares: estrategias teórico-metodológicas para su abordaje. XI Jornadas de Sociología. Buenos Aires: Universidad de Buenos Aires. Recuperado de http://cdsa.aacademica.org/000-061/782

Vargas-Evaristo, S. (2006). El papel de los niños trabajadores en el contexto familiar. El caso de migrantes indígenas asentados en el valle de San Quintín, B. C. Papeles de Población, (12)48, 227-245. Recuperado de http://www. redalyc.org/pdf/112/11204811.pdf

Velasco-Ortiz, L. (2013). Escuela y reproducción social de familias migrantes: hijos e hijas de jornaleros indígenas en el noroeste mexicano. Estudios Demo- 
gráficos y Urbanos, 28(1)(82), 189-218. Recuperado de http://www. redalyc. $\mathrm{org} / \mathrm{pdf} / 312 / 31230009006 . \mathrm{pdf}$

Yurén-Camarena, T., Rojas-Mendoza, A., de la Cruz-Reyes, M., Espinosa-Meléndez, J., y Escalante-Ferrer, A. E. (2011). Cuando la justicia falla por simpleza... Análisis de políticas y trayectorias de escolaridad en el caso de la población jornalera agrícola. Sinéctica, Revista Electrónica de Educación, 37, 1-17. Recuperado de http://www.scielo.org.mx/pdf/sine/n37/n37a4.pdf 